\title{
Electrophysiology of nociception: understanding of signaling pathways forms a basis for potential treatment
}

\author{
Lubica Lacinova ${ }^{1}$ \\ Published online: 28 February 2022 \\ (๐) The Author(s), under exclusive licence to Springer-Verlag GmbH Germany, part of Springer Nature 2022
}

Damage of either in peripheral, visceral, and deep tissues results in an unpleasant feeling recognized as a pain. Feeling of a pain is a result of a processing of a complex nociceptive signal transmitted from the point of damage into central nervous system. While nociception denotes an objective physiological process of transmitting noxious stimuli into the brain, pain represents a subjective interpretation of a sensory event processed by the brain. Nociceptive signaling starts with any stimulus having potential to cause tissue damage, e.g., mechanical pressure, high or low temperature, aggressive chemicals like acids or alkalis. Pathological processes like inflammation also involve release of chemical messengers, e.g., cytokines which are capable to initiate nociception. These noxious stimuli are converted at the endings of specific sensory neurons-nociceptors-into electrical signal. Several types of nociceptors differing by myelination, fiber diameter, and conduction velocity are known. Nociceptive signal is being carried along their axons through dorsal root ganglions, and further it is transmitted via spinothalamic tract or via trigeminal ganglion into the various brain areas.

Nociceptive signal initiated by an acute injury has a defensive function. On the other hand, serious chronic diseases like diabetes, cancer, inflammatory diseases, and various nerve injuries results in long lasting chronic pain developed due to sensitization of nociceptors which results in allodynia (the lowered threshold for pain perception) or hyperalgesia (exaggerated pain sensation caused by relatively weak pain stimuli) which negatively affects the quality of life. Chronic pain is one of the most common reasons why adults seek medical care, and therefore it has a significant impact on society and individuals. The Global Burden of

Lubica Lacinova

lubica.lacinova@savba.sk

1 Center of Biosciences, Institute of Molecular Physiology and Genetics, Slovak Academy of Sciences, Bratislava, Slovakia
Disease Study 2016 [2] confirmed that pain and pain-related diseases represent the leading cause of disability and disease burden globally. Detailed understanding of molecular mechanisms of raise and spread of nociceptive signal will allow design of effective therapies.

In this special issue of Pflügers Archive - European Journal of Physiology on electrophysiology of nociception 5 reviews and 4 original articles offer a complex view on various aspects of origin, spread, and processing of nociceptive signals. Peripheral endings of nociceptors express a variety of ion channels whose activation by noxious stimuli leads to membrane depolarization and eventually to activation of an action potential carrying the nociceptive signal into the central nervous system. Two major classes of these ion channels represent TRP (transient receptor potential) channels and PIEZO channels, who's discovery was honored by the Nobel price 2021 for physiology and medicine [9]. Individual members of the family of TRP channels can convert various stimuli into an electrical signal, i.e., an action potential: heat, cold, pungent plant compounds, chemicals released during inflammatory process, and mechanical stimuli like pressure and stretch. PIEZO channels are more specialized being activated by mechanical stimuli, i.e., pressure and stretch. Once activated e.g., due to peripheral nerve injury, action potentials spread along nociceptive neuron fibers. Multiple voltage-dependent ion channels, $\mathrm{Na}_{\mathrm{V}}$ and $\mathrm{K}_{\mathrm{V}} 7$ channels as well as voltage- and ligand-gated $\mathrm{HCN}$ channels, participate in this process and thus represent a potential peripheral therapeutic target for the treatment of positive symptoms of neuropathic pain [10]. Cell bodies of nociceptors are located in the dorsal root ganglia (DRG) or trigeminal ganglia. These are even more diverse in their electrophysiological properties than the nociceptors themselves. Systematic review of available literature identified five grouping criteria for classification of rat DRGs. No sufficient number of data are available for human DRG neurons; however, it is clear that findings from rat neurons cannot be transferred offhand to the human system [8]. Further functional data on human 
sensory neurons are needed to facilitate progress in development of pain treatment.

Drugs used for management of severe neuropathic and/or chronic pain often target voltage-gated ion channels. While sodium channels have a decisive role in initiation of an AP firing and potassium channel determine their repolarization phase, voltage-gated calcium channel modulate the excitability of nociceptors in a complex way. Prominent role in nociception play $\mathrm{Ca}_{\mathrm{V}} 2.2$ and $\mathrm{Ca}_{\mathrm{V}} 3.2$ channels [7]. These channels also represent a popular target for development of new analgesics.

Various modulatory pathways affecting voltage-dependent ion channels involved in the nociception were described. The receptor for activated C kinase 1 (Rack-1) has been implicated in neuropathic pain. Rack-1 forms a complex with the $\mathrm{Ca}_{\mathrm{v}} 3.2$ channel and suppresses whole cell current density in the absence of protein kinase C $\beta I I$ [4]. Endogenous amino acid l-cysteine potentiated calcium current through the $\mathrm{Ca}_{\mathrm{v}} 2.3$ channel likely by chelating the trace metals that tonically inhibit the channel [5]. The system L-neutral amino acid transporter Slc7a5 (Lat1) not only serves as a transporter for anti-allodynic gabapentinoid drugs but also binds to $\mathrm{Na}_{\mathrm{V}} 1.7, \mathrm{~K}_{\mathrm{V}} 1.1$, and $\mathrm{K}_{\mathrm{V}} 1.2$ channels, which are implicated in nociception and chronic pain. It was demonstrated that Slc7a5 is dysregulated in chronic neuropathic pain and can be targeted in treatment of hyperalgesia [6].

Role of long non coding RNAs (lncRNA) in modulation of various proteins is a rapidly growing field of research. Ion channels implicated in nociception, e.g., TRPV1, $\mathrm{Na}_{\mathrm{V}}, \mathrm{K}_{\mathrm{V}}$, and $\mathrm{P} 2 \mathrm{X}$ channels, are among lcnRNAs targets [3]. Research on the role of lncRNAs involved in nociception opens a new and promising direction for development of potential pain treatment.

Physiological processes in an organism are interdependent in very complex way. It was shown that an unavoidable fear stimuli, e.g., when a prey faces a threat, leads to immobility defensive reaction associated with a suppressed pain sensation, which increase the chances of survival. These two reactions are coordinated by the ventrolateral periaqueductal gray matter (vlPAG) but are not mutually dependent [1]. Nevertheless, this observation identifies vlPAG as another potential target for antinociceptive treatment.

\section{References}

1. de Mello Rosa GH, Ullah F, de Paiva YB, da Silva JA, Branco LGS, Corrado AP, Medeiros P, Coimbra NC, Biagioni AF (2022) Ventrolateral periaqueductal gray matter integrative system of defense and antinociception. Pflugers Arch. https://doi.org/10. 1007/s00424-022-02672-0

2. GBD (2016) Disease and Injury Incidence and Prevalence Collaborators: (2017) Global, regional, and national incidence, prevalence, and years lived with disability for 328 diseases and injuries for 195 countries, 1990-2016: a systematic analysis for the Global Burden of Disease Study 2016. Lancet 390:1211-1259. https:// doi.org/10.1016/S0140-6736(17)32154-2

3. Felix R, Muñoz-Herrera D, Corzo-Lopez A, Fernandez-Gallardo M, Leyva-Leyva M, González-Ramírez R, Sandoval A (2022) Ion Channel Long Non-coding RNAs in Neuropathic Pain. Pflugers Arch

4. Gandini MA, Souza IA, Khullar A, Gambeta E, Zamponi GW (2021) Regulation of CaV3.2 channels by the receptor for activated $\mathrm{C}$ kinase 1 (Rack-1). Pflugers Arch. https://doi.org/10.1007/ s00424-021-02631-1

5. Ghodsi SM, Walz M, Schneider T, Todorovic SM (2022) L-cysteine modulates visceral nociception mediated by the Cav3.2 R-type calcium channels. Pflugers Arch

6. Goins AE, Gomez K, Ran D, Afaghpour-Becklund M, Khanna R, Alles SRA (2022) Neuronal allodynic mechanisms of Slc7a5 (LAT1) in the spared nerve injury rodent model of neuropathic pain. Pflugers Arch. https://doi.org/10.1007/s00424-021-02653-9

7. Hoppanova L, Lacinova L (2022) Voltage-dependent CaV3.2 and CaV2.2 channels in nociceptive pathways. Pflugers Arch. https:// doi.org/10.1007/s00424-022-02666-y

8. Korner J, Lampert A (2022) Functional subgroups of rat and human sensory neurons: a systematic review of electrophysiological properties. Pflugers Arch. https://doi.org/10.1007/ s00424-021-02656-6

9. Reeh PW, Fischer MJM (2022) Nobel somatosensations and pain. Pflugers Arch. https://doi.org/10.1007/s00424-022-02667-x

10. Roza C, Bernal L (2022) Electrophysiological characterization of ectopic spontaneous discharge in axotomized and intact fibers upon nerve transection: a role in spontaneous pain? Pflugers Arch. https://doi.org/10.1007/s00424-021-02655-7

Publisher's note Springer Nature remains neutral with regard to jurisdictional claims in published maps and institutional affiliations. 